\title{
Changes in the activity of ovine blood-derived macrophages stimulated with antimicrobial peptide extract (AMP) or platelet-rich plasma (PRP)
}

\author{
Joanna Wessely-Szponder ${ }^{1}$, Tomasz Szponder ${ }^{2}$, Ryszard Bobowiec $^{1}$, Joanna Michalska ${ }^{1}$ \\ ${ }^{1}$ Department of Pathophysiology, Chair of Preclinical Veterinary Sciences, \\ ${ }^{2}$ Department and Clinic of Animal Surgery, Faculty of Veterinary Medicine, \\ University of Life Sciences, 20-033 Lublin, Poland \\ tomszpon@op.pl
}

Received: September 5, 2018 Accepted: March 25, 2019

\begin{abstract}
Introduction: Antimicrobial peptides (AMP) are a large group of innate immune effectors, which apart from antimicrobial activity show immunomodulative properties. Platelet-rich plasma (PRP) is a source of autologous growth factors and is used for stimulation of bone and soft tissue healing. The purpose of this study was to assess the influence of PRP and AMP extract on ovine monocyte-derived macrophage cultures. Material and Methods: The study was conducted on ovine macrophages (Mfs) previously stimulated with LPS or dexamethasone and then with preparations of PRP or AMP. Following activation of the Mfs their morphological and functional features were assessed. Results: The study revealed pro-inflammatory influence of both examined preparations on Mfs cultures on the basis of morphology, ROS generation and arginase activity. Both preparations enhanced the pro-inflammatory response of cultured Mfs. Conclusion: This activity may intensify the antimicrobial action of Mfs, however, in cases of excessive and prolonged inflammation the use of these preparations should be limited.
\end{abstract}

Keywords: monocyte-derived macrophages, antimicrobial peptides, platelet-rich plasma.

\section{Introduction}

Antimicrobial peptides (AMP), also known as host defence peptides, are important components of innate immunity. As has been stated, some of these peptides have immunoregulatory activity in addition to their potent antimicrobial action. AMP display highly diverse sequences and biological functions. In mammals, they are divided into two main groups: cathelicidins and defensins (19). Cathelicidins are the host defence peptides which show antimicrobial activity together with multiple functions relating to innate immunity and tissue repair $(2,13)$. Mammalian defensins are a large family of cysteine-rich antimicrobial peptides divided into three subfamilies: $\alpha-, \beta$-, and $\theta$-defensins (19).

Some mixtures of natural preparations based on antimicrobial peptides have been evaluated as therapeutic means for fighting infections and strengthening host defence mechanisms (2). As noted previously, a rabbit AMP extract was evaluated which consisted of a $15 \mathrm{kDa}$ antimicrobial peptide, cathelinlike fragments, CAP 18, and rabbit defensins NP-1, NP-2, NP-3a, NP-3b, NP-4, and NP-5, and may be regarded as a promising new therapeutic approach for regulation of the intensity of the inflammatory process with the potential for immunomodulation (24).

Platelet-rich plasma (PRP) has been used for several years during surgical procedures to enhance peri-implant soft tissue and bone healing and has been applied for regeneration of bone, cartilage, and ligaments. The main rationale for the use of PRP stems from the growth factors released from platelet $\alpha$-granules, including platelet-derived growth factor (PDGF), transforming growth factor (TGF), vascular endothelial growth factor (VEGF), and insulin-like growth factor (IGF) (20). All these growth factors were detected previously in preparations of PRP from ovine blood together with antimicrobial peptide (PF4), which enhances their antimicrobial properties (23). These 
antimicrobial properties of platelet components would be best taken advantage of producing a combined neutrophil and platelet extract to maximise their activity (2).

Macrophages (Mfs) are the main effectors and regulators of innate inflammation. The key functions of these cells are phagocytosis, killing of invading pathogens, and release of inflammatory mediators; they also play a role in healing processes (9). Owing to Mf plasticity, there are possibilities to shift them from being pro-inflammatory to being regulatory suppressive cells (9). The study of the Mf response to stimulation with two different blood-derived agents, AMP extract and PRP could shed new light on the application of alternative substances in enhancement of tissue healing and infection fighting. Therefore, the aim of this study was to assess the influence of autologous ovine PRP and rabbit AMP extracts on ovine monocyte-derived Mf (MDM) populations, in respect of their proinflammatory or tissue repair properties.

\section{Material and Methods}

Preparation of the neutrophil crude extract of rabbit AMP. In the first step of the experiment neutrophils were purified from $2-3 \mathrm{~mL}$ portions of blood obtained from six healthy New Zealand White rabbits in accordance with animal protection regulations. After red blood cell lysis with $0.83 \%$ ammonium chloride solution followed by differential centrifugation, the final polymorphonuclear leukocyte cells were then homogenised to release the neutrophil granules. These granules were centrifuged at 25,000 $\times \mathrm{g}$ for $40 \mathrm{~min}$ at $4^{\circ} \mathrm{C}$, then acid extraction with $10 \%$ acetic acid was performed and insoluble material was sedimented $\left(25,000 \times \mathrm{g}, 20 \mathrm{~min}, 4^{\circ} \mathrm{C}\right)$. The supernatant containing antimicrobial peptides was considered AMP extract and lyophilised. Portions of $40 \mu \mathrm{g} / \mathrm{mL}$ of this extract were dissolved in PBS, and then used for stimulation of macrophage cultures (25).

Preparation of PRP from sheep blood. Blood samples of $8.5 \mathrm{~mL}$ were collected from the jugular vein of Polish Lowland sheep into a monovette containing citrate phosphate, dextrose, and adenosine (CPDA) from a PRP kit (Curasan AG, Germany). A two-step procedure was applied, in which first the blood samples were centrifuged at $160 \times g$ for $20 \mathrm{~min}$ at room temperature to separate the plasma containing the platelets. Then the plasma fraction was collected and centrifuged $(400 \times g, 15 \mathrm{~min})$. The top layer was discarded and the remaining volume was platelet-rich plasma (23).

Monocyte isolation and generation of polarised monocyte-derived macrophages from sheep blood. Peripheral blood mononuclear cells were separated from ovine blood by gradient centrifugation with Lymphoprep (Nycomed, Norway) as the density gradient medium. The obtained cells were plated at a density of $1.0 \times 10^{6}$ cells/mL onto 96 -well flat-bottom tissue culture plates (Nunc, USA) and cultured at $37^{\circ} \mathrm{C}$ in $5 \% \mathrm{CO}_{2}$ for $24 \mathrm{~h}$ in Dulbecco's modified Eagle's medium (DMEM) with 10\% bovine calf serum (BCS) (Biomed, Poland). The adherent cells were cultured for an additional $48 \mathrm{~h}$ to allow monocytes to mature to functional macrophages (10). Then, polarisation of cultures began by changing media to one of three variants supplemented as follows: without stimulators, for generation of BCS Mfs as a control; with addition of $1 \mu \mathrm{g} / \mathrm{mL}$ LPS from E. coli serotype 055:B5 (SigmaAldrich, Poland), for generation of M1 (described as LPS); and with dexamethasone (DEX) at a concentration of $100 \mathrm{nM}$ for generation of M2 (22, 25). After polarisation Mfs were additionally stimulated with one of two different stimulators: PRP or AMP. Addition of PRP generated cultures marked as PRP, LPS+PRP, or DEX+PRP, according to the previous stimulation. Addition of AMP $(40 \mu \mathrm{g} / \mathrm{mL}$ of rabbit AMP extract reconstructed from lyophilisate) generated cultures marked as AMP, LPS+AMP, and DEX+AMP likewise corresponding to the previous stimulator used. On every subsequent day of culture the cells were subjected to microscopic analysis of their morphology using an inverted microscope. The functional analysis was conducted $24 \mathrm{~h}$ (time $1-\mathrm{T} 1)$ and $48 \mathrm{~h}$ after stimulation (time $2-\mathrm{T} 2$ ).

In vitro functional analysis. Nitric oxide (NO) concentration was assessed using the Griess method. Briefly, an equal volume of culture supernatant and Griess reagent $(0.1 \%$ N-(1-naphthyl)ethylenediamine dihydrochloride $1 \%$ sulphanilamide and $\left.2.5 \% \mathrm{H}_{3} \mathrm{PO}_{4}\right)$ were mixed and incubated at room temperature for 20 min and then absorbance was measured. The obtained values were expressed as a concentration of nitrite (the stable breakdown product of NO, which accumulates in the medium). Conversion of absorbance to micromoles $(\mu \mathrm{M})$ was calculated from a $\mathrm{NaNO}_{2}$ standard curve (25).

Superoxide production was measured using a method given previously (24). In brief, the media obtained from cultures of Mfs with different stimulators were incubated with $0.1 \%$ nitroblue tetrazolium (SigmaAldrich, Poland) solution at room temperature for $15 \mathrm{~min}$ and then absorbance was read. The amounts of the superoxide in nanomoles (nMs) were calculated using the extinction coefficient $21.1 \mathrm{nM}$.

Arginase activity was assessed by measuring the concentration of urea generated by arginase-dependent hydrolysis of L-arginine. Macrophages were lysed with $0.1 \%$ Triton $\mathrm{X}-100$ and the cell lysates were incubated with $50 \mu \mathrm{L}$ of $25 \mathrm{mM}$ Tris- $\mathrm{HCl}$ and $10 \mu \mathrm{L}$ of $10 \mathrm{mM}$ $\mathrm{MnCl}_{2}$. Then, after thermal activation of the enzyme $\left(10 \mathrm{~min}\right.$ at $\left.55^{\circ} \mathrm{C}\right)$, L-Arginine $(0.5 \mathrm{M})$ was hydrolysed at $37^{\circ} \mathrm{C}$ for $120 \mathrm{~min}$. Next, the reaction was stopped with $400 \mu \mathrm{L}$ of $\mathrm{H}_{2} \mathrm{SO}_{4} / \mathrm{H}_{3} \mathrm{PO}_{4} / \mathrm{H}_{2} \mathrm{O}(1 / 3 / 7 \mathrm{vol} / \mathrm{vol} / \mathrm{vol})$. The urea concentration was measured after addition of $40 \mu \mathrm{L}$ of $\alpha$-isonitrosopropiophenone (Sigma-Aldrich, Poland) and heating at $100^{\circ} \mathrm{C}$ for $40 \mathrm{~min}$. The activity of arginase was determined on the basis of urea 
formation estimated by a comparison with a standard curve (7).

Statistical analysis. At least three independent experiments in four replications were performed. All data are expressed as the mean \pm standard error (SE) for continuous variables. Significance was identified by one-way ANOVA using Statistica 13.1 (StatSoft, Poland) followed by Tukey's test. Differences were considered statistically significant when $\mathrm{P}$ was $<0.05$.

\section{Results}

Changes in morphology of MDM after stimulation with different factors are shown in Fig. 1. Unstimulated

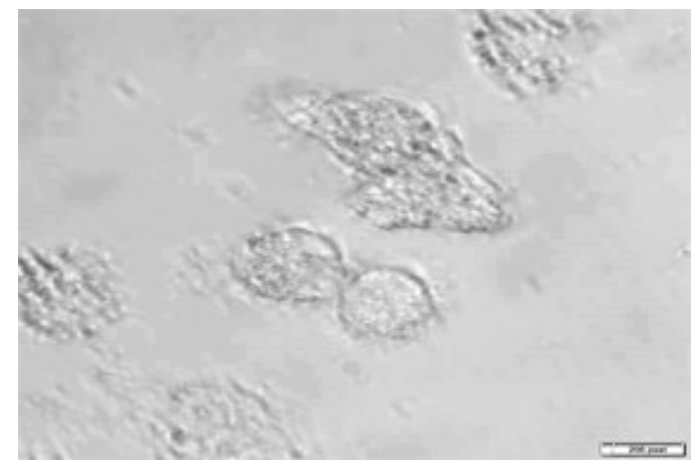

(A)

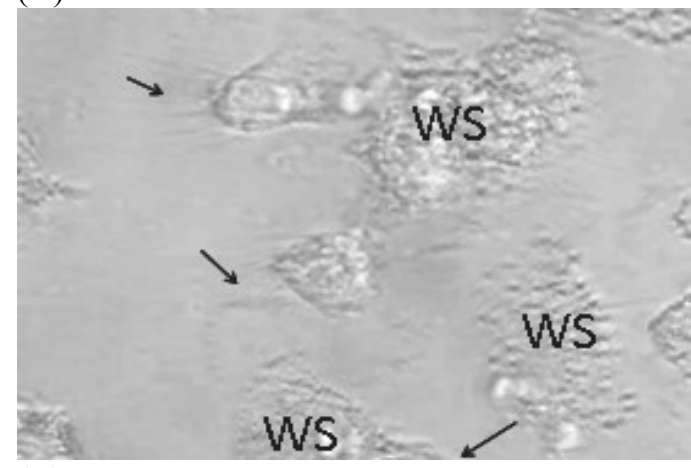

(C)

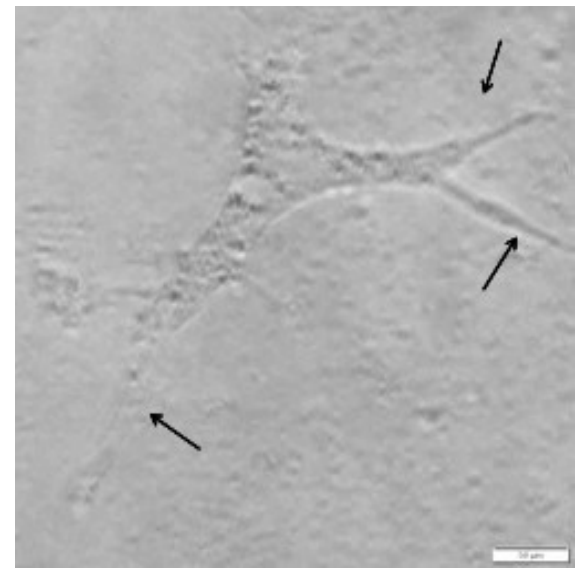

(E)
MDMs (cultured for $72 \mathrm{~h}$ with DMEM enriched with $10 \%$ calf serum) were classified as BCS (Fig. 1A). The addition of LPS (Fig. 1B) generated dendric-like MDMs with large filopodia. Treatment of MDM cultures with AMP extract resulted in well-spread cells with multiple filopodia (Fig. 1C). In contrast, addition of dexamethasone induced an increase in rounded cells (Fig. 1D). After stimulation with PRP, MDMs adopted a dendric-like morphology with long filopodia (Fig. 1E), whereas, after previous stimulation with LPS, addition of PRP caused cells to be well spread and dendric-like with large filopodia (Fig. 1F).

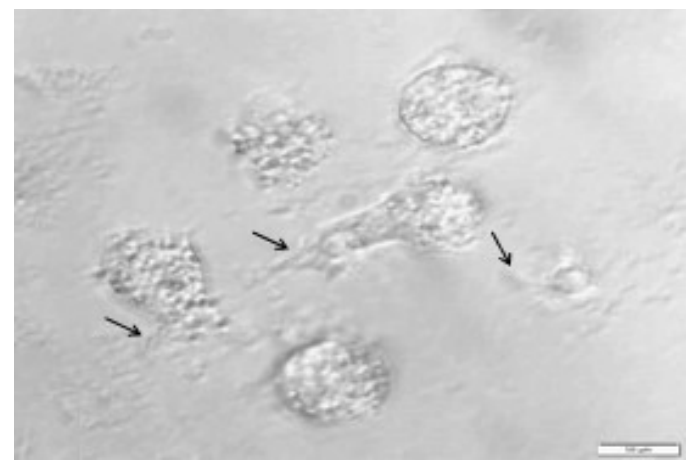

(B)

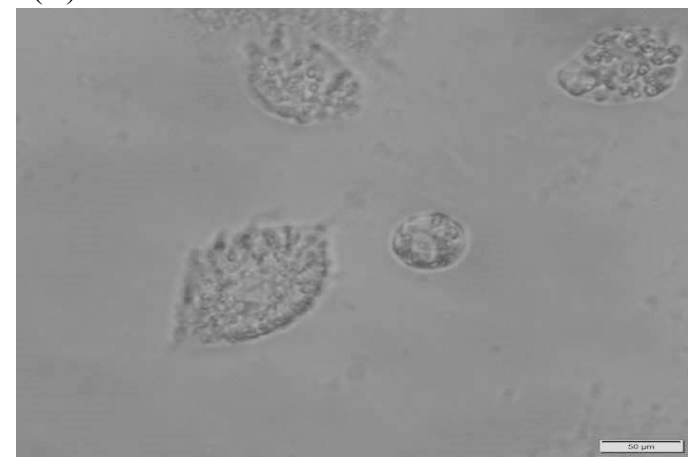

(D)

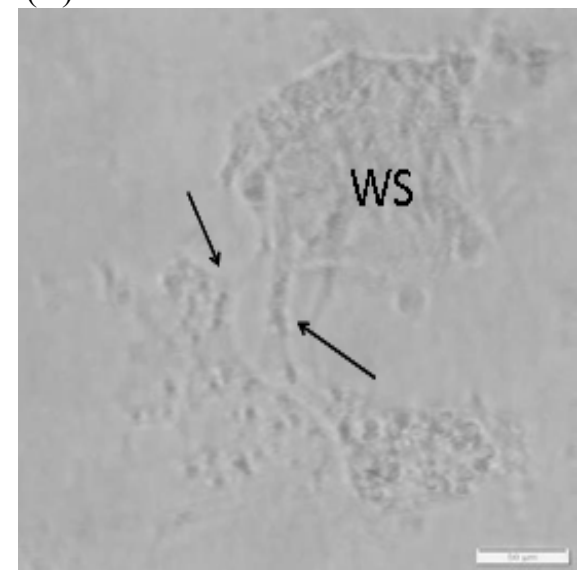

(F)

Fig. 1. Changes in morphology of MDM after stimulation with different factors. A - unstimulated MDMs (cultured for $72 \mathrm{~h}$ with DMEM enriched with $10 \%$ calf serum) classified as BCS. B - M1 Mfs after addition of LPS (black arrows indicate large filopodia). C - Mfs after addition of AMP extract, well-spread cells with multiple filopodia (black arrows). D - Mfs culture after addition of dexamethasone (DEX). E - stimulation of Mfs with PRP, cells with long filopodia (black arrows). F - Mfs culture after previous stimulation with LPS and addition of PRP, well-spread cells (WS) and dendric-like cells with large filopodia (black arrows). The morphology of Mfs was assessed by phase-contrast microscopy. Original magnification $\times 40$ (CK-40, Olympus, Japan). Representative images are shown from $\mathrm{n}=6$ replicates 
As expected, Mfs under the proinflammatory stimulation with LPS showed M1 features in the form of increased NO (Fig. 2) and superoxide generation (Fig. 3) and decreased arginase activity in comparison with BCS Mfs (Fig. 4). Addition of DEX, in turn, evoked an anti-inflammatory response representative of M2 Mfs, presenting as a decrease in ROS generation at both T1 and T2 (Fig. 2 and Fig. 3). The ratio of urea generation to nitrite generation measured against that of BCS Mfs fell significantly $(\mathrm{P}<0.05)$ in the LPS group and rose significantly in the DEX group (Fig. 5).

A significant increase in NO generation from 3.5 $\pm 0.4 \mu \mathrm{M} / 10^{6}$ cells in BCS Mfs to $5.2 \pm 0.29 \mu \mathrm{M} / 10^{6}$ cells in the PRP group and to $5.64 \pm 0.4 \mu \mathrm{M} / 10^{6}$ cells in the LPS + PRP group was detected in the second measurement (Fig. 2). Stimulation with PRP caused generation of more superoxide, especially at T1, and in cultures previously stimulated with LPS this response was more pronounced (Fig. 3). Arginase activity was diminished in all groups stimulated with PRP (Fig. 4). The ratio of urea generation to nitrite generation was significantly $(\mathrm{P}<0.05)$ lower than that in the BCS group and near the value obtained for M1 Mfs (Fig. 5).

Stimulation of $\mathrm{Mf}$ cultures with AMP significantly $(\mathrm{P}<0.05)$ depressed superoxide production at T2 in all cultures (Fig. 3). On the other hand, stimulation of Mfs with AMP caused greater NO generation, especially at T2 (Fig. 2). Stimulation of Mf cultures with AMP alone inhibited arginase activity, however, previous supplementation with other stimulators weakened this response (Fig. 4). In all groups stimulated with AMP the ratio of urea generation to nitrite generation was significantly lower than that in the BCS group, with the highest value seen in the DEX + AMP group (Fig. 5).

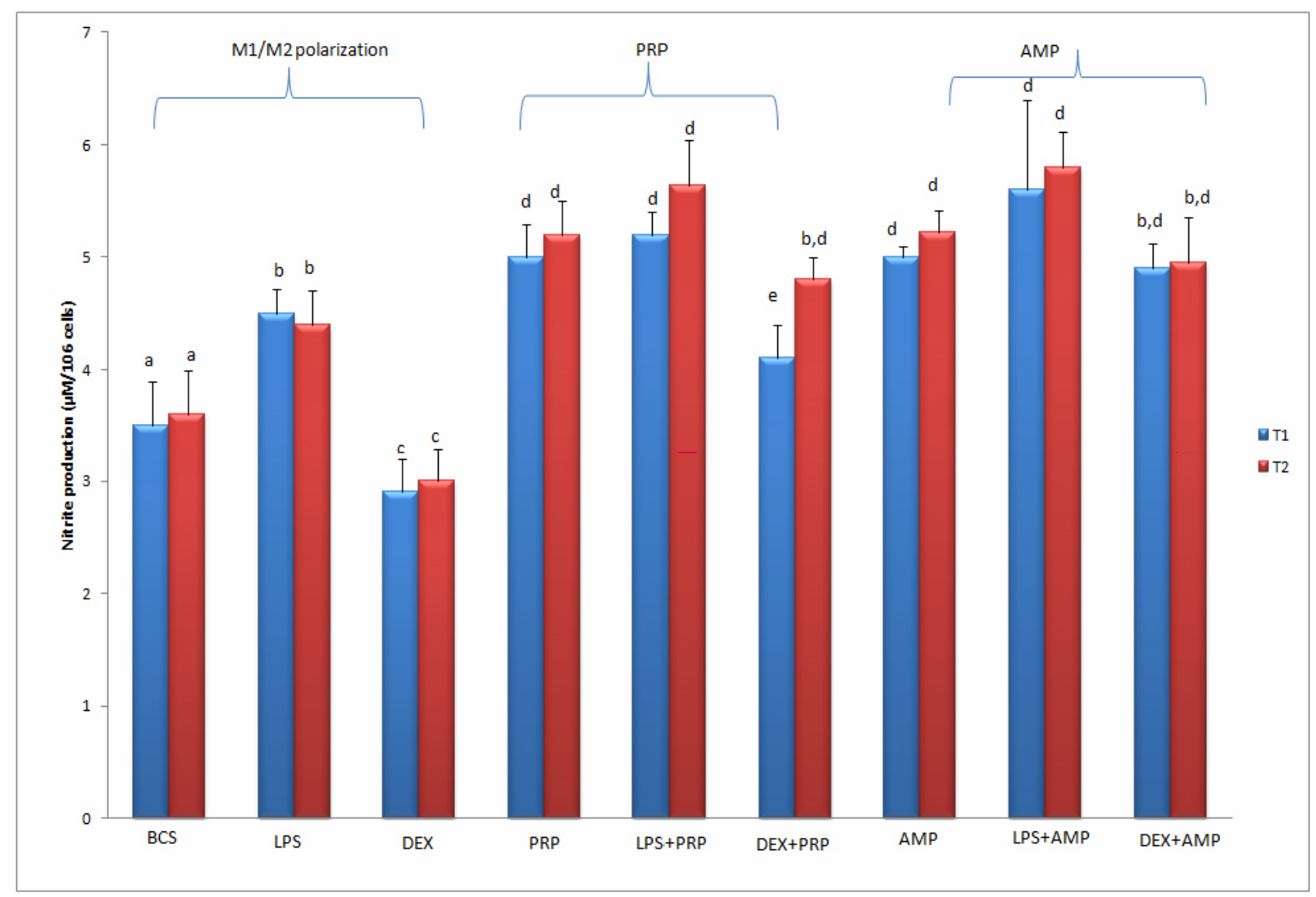

Fig. 2. Nitric oxide generation by Mfs after $24 \mathrm{~h}$ and $48 \mathrm{~h}$ stimulation as follows: BCS - cultures stimulated only with DMEM with $10 \%$ BCS, LPS - cultures stimulated with $1 \mu \mathrm{g} / \mathrm{mL}$ LPS, DEX - cultures stimulated with $100 \mathrm{nM}$ of dexamethasone, PRP - cultures stimulated with PRP, LPS + PRP - cultures stimulated with PRP after previous incubation with LPS, DEX + PRP - cultures stimulated with PRP after previous incubation with dexamethasone, AMP - cultures stimulated with AMP extract, LPS + AMP - cultures stimulated with AMP extract after previous incubation with LPS, DEX + AMP - cultures stimulated with AMP extract after previous incubation with dexamethasone. Values are means \pm SE obtained from each separate experiment conducted on three independent cultures in four replications. Mean values marked with different letters differ statistically $(\mathrm{P}<0.05)$ 


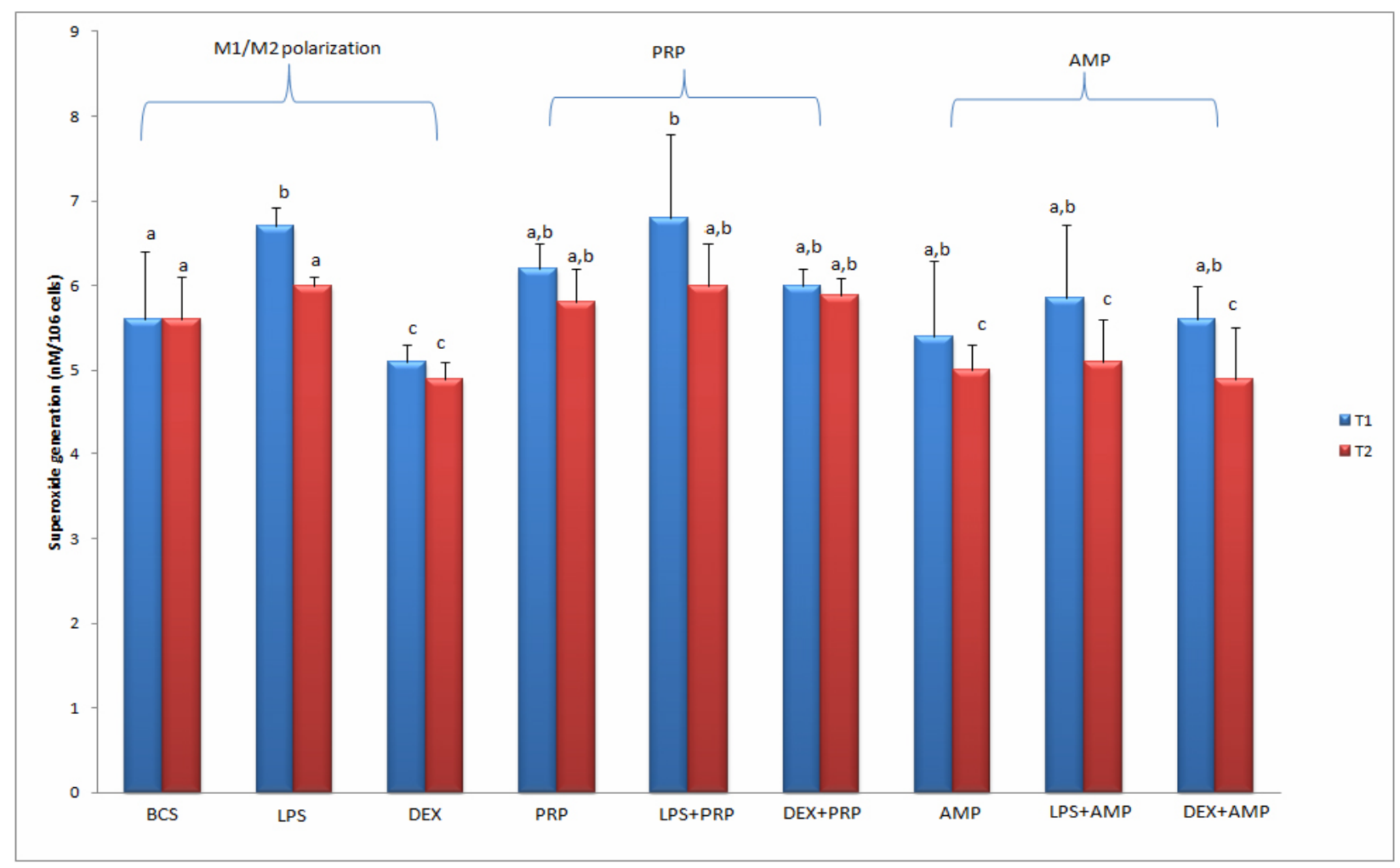

Fig. 3. Superoxide generation by Mfs after $24 \mathrm{~h}$ and $48 \mathrm{~h}$ stimulation. For abbreviations see footnote to Fig. 2. Values are means $\pm \mathrm{SE}$ obtained from each separate experiment conducted on three independent cultures in four replications. Mean values marked with different letters differ statistically $(\mathrm{P}<0.05)$

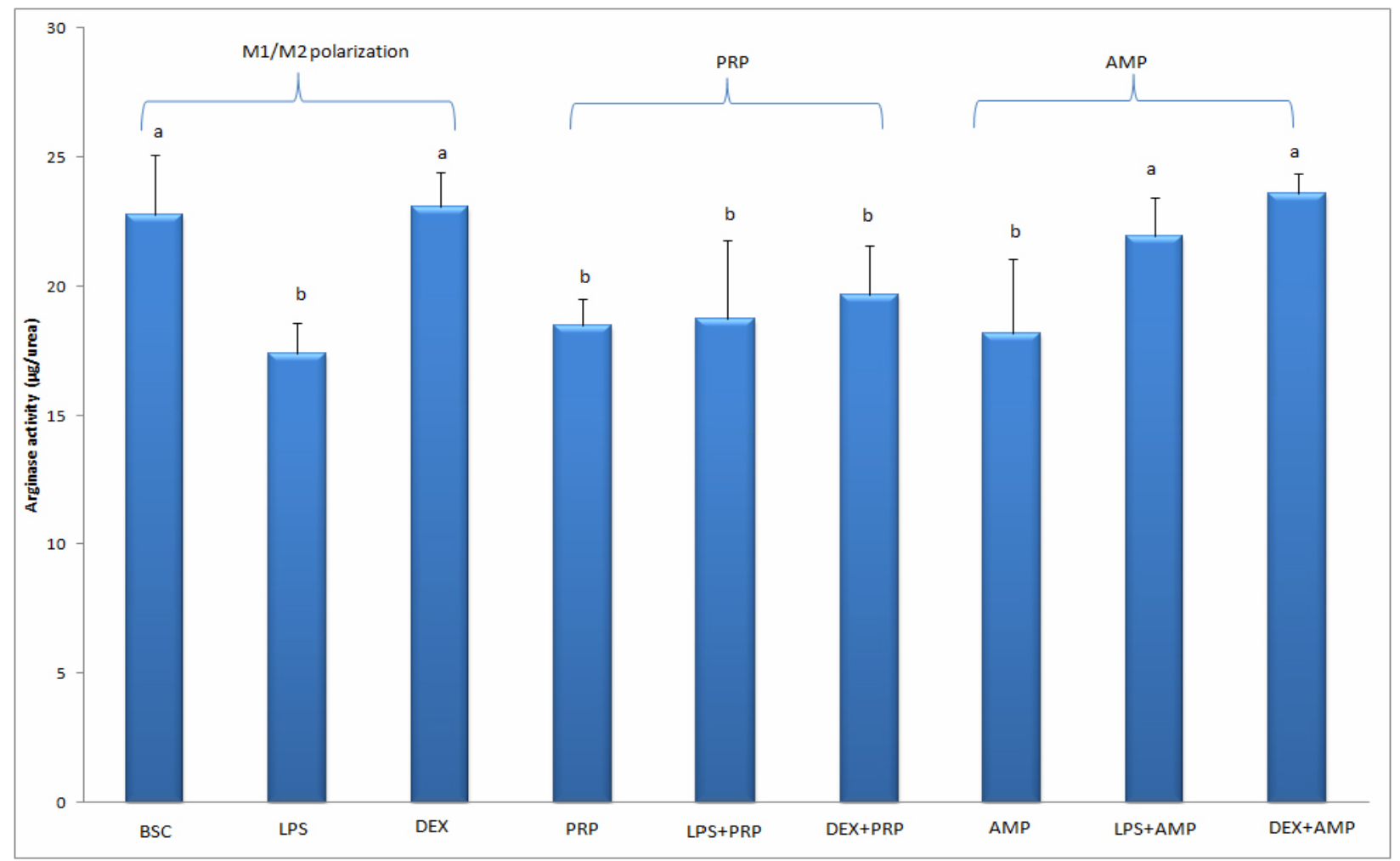

Fig. 4. The arginase activity of differentiated Mfs cultures. For abbreviations see footnote to Fig. 2. Values are means \pm SE obtained from each separate experiment conducted on three independent cultures in four replications. Mean values marked with different letters differ statistically $(\mathrm{P}<0.05)$ 


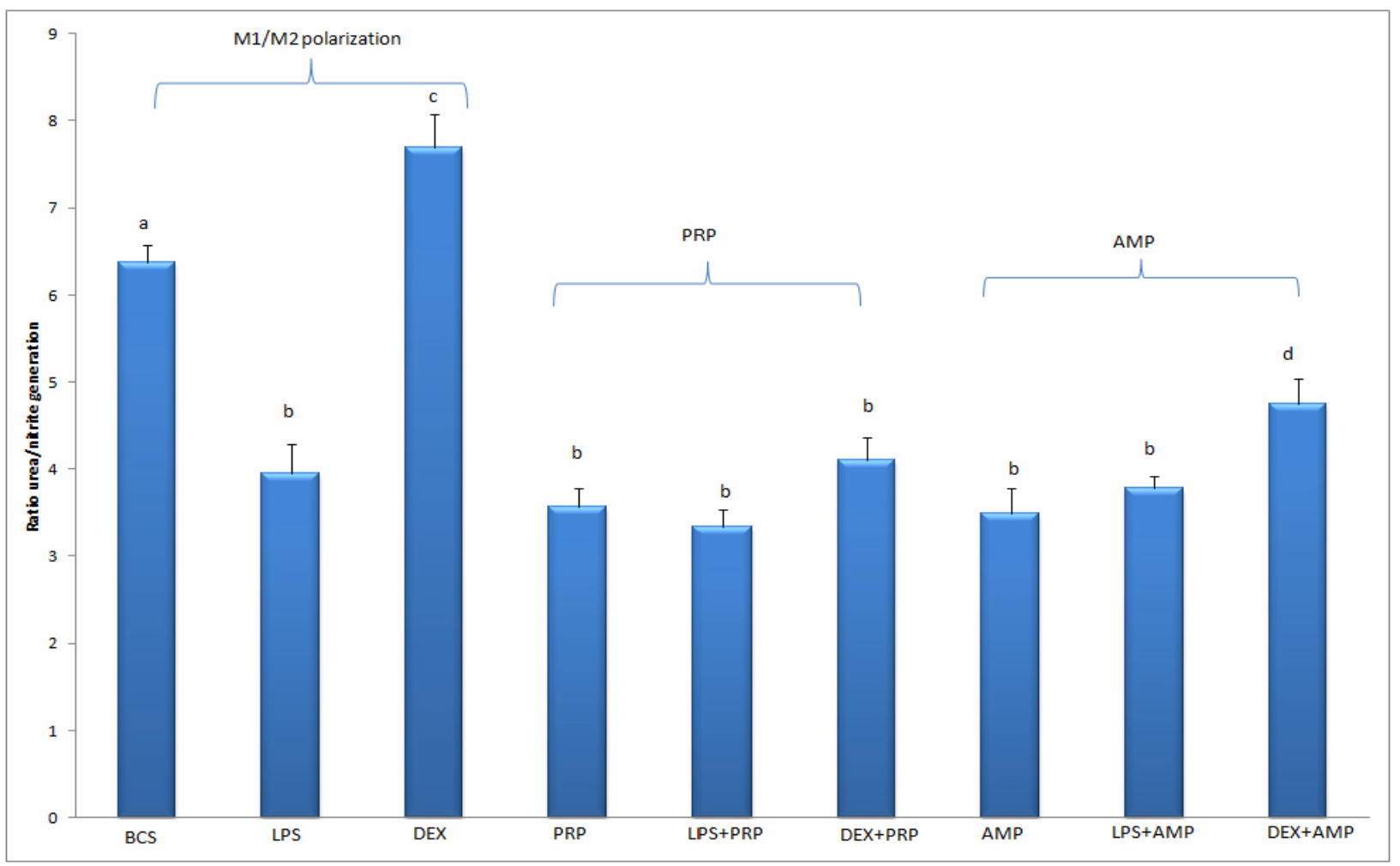

Fig. 5. The ratio of urea generation to nitrogen generation of differentiated Mfs cultures. For abbreviations see footnote to Fig. 2. Values are means \pm SE obtained from each separate experiment conducted on three independent cultures in four replications. Mean values marked with different letters differ statistically $(\mathrm{P}<0.05)$

\section{Discussion}

In the first step of this study, we demonstrated that MDM morphology and function after polarisation differentiated to pro-inflammatory or repair phenotype according to the stimulator used. During tissue injury or infection the first-responder Mf usually exhibits a pro-inflammatory phenotype and secretes proinflammatory cytokines, superoxide, and NO, for activation of various mechanisms, which contribute to the elimination of invading organisms (15). However, the previous study by Campbell et al. (5) revealed that Mf phenotype is temporally regulated during wound healing such that M1 are present at early stages and M2 predominate during later stages of inflammation, with increased arginase activity as one of the markers. Local Mf polarisation has a great impact on healing, however, an excessive inflammatory response is a major problem in pathological inflammation (18).

In our experiment, after stimulation with LPS Mfs adopted a fully differentiated ameboid morphology characteristic of M1. This feature of Mfs was previously described by Ploeger et al. (17). On the other hand, the rounded cells previously noted by Giles et al. (11) after treatment with glucocorticoids were also observed in our study after stimulation of Mfs with DEX (18). The addition of AMP changed Mf morphology; they transformed into well-spread cells with multiple filopodia, as described previously $(24,25)$. After contact with PRP, Mfs changed towards the pro-inflammatory response both in cultures after previous stimulation with LPS and in those without this stimulation.

Addition of LPS produced more abundant superoxide in Mf culture, as observed previously, and this response was involved in polarisation of Mfs towards M1. Contrastingly, supplementation with DEX resulted in M2 Mfs with augmented arginase activity and decreased free radical generation. This response noted by us was previously also described by other authors in the case of M2c Mfs $(6,12,14)$.

In the further part of the study we demonstrated a significant decrease in superoxide generation in cultures stimulated after $48 \mathrm{~h}$ with AMP. Previously, it was seen that rabbit AMP extract diminished superoxide level in culture of rabbit MDMs (10), and a similar effect was also mentioned by Agier et al. (1). The stimulation of Mfs with PRP resulted in more superoxide generation, with the highest response evident after priming with LPS. Some authors mentioned a pro-inflammatory response of $\mathrm{Mfs}$ after stimulation with PRP (8), whereas others suggested Mf differentiation towards a repair profile (3).

Our experiment revealed more powerful generation of NO in cultures stimulated with PRP alone or in combination with LPS (LPS + PRP) or DEX (DEX + PRP). Similarly, all Mf cultures treated with AMP alone or with other stimulators showed uplifted production of NO in comparison with unstimulated BCS Mfs. This response indicates a pro-inflammatory 
phenotype of Mfs, as described previously by Mantovani et al. (12).

Arginase activity significantly $(\mathrm{P}<0.05)$ decreased after addition of LPS, PRP, LPS+PRP or AMP to MF cultures, whereas previous stimulation with DEX caused a slight increase in arginase activity. Additionally, the urea to nitrogen ratio came down in all groups with the exception of the cultures stimulated with DEX alone where increased arginase activity was detected. The balance between inducible nitric oxide synthase (iNOS) and arginase activity, which both compete for the common product L-arginine, is tightly regulated during repair, and decreased arginase activity is observed in human non-healing wounds, indicating its potential importance for healing. Moreover, an absence of iNOS delays healing, whereas iNOS upregulation correlates with faster healing (4).

According to Scull et al. (26), the interaction of human Mfs with autologous platelets results in scavenger-receptor-mediated platelet uptake and enhancement of LPS-induced cytokine secretion. Conversely, the research of Arrepol et al. (3) revealed that human autologous PRP programmed Mfs toward a repair profile. Our study did not confirm this effect from what was seen of ovine platelet-Mf interactions. Our results showed pro-inflammatory response to autologous PRP with a slight rise in arginase (as a marker) in comparison with LPS-stimulated M1. The previous work of Czakai et al. (8) indicated that human PRP intensified the antimicrobial activity against pathogens generated by human monocyte-derived Mfs as well as the pro-inflammatory Mf response to PRP alone.

During a local inflammatory process, activated platelets intensified the Mf pro-inflammatory response. However, Scull et al. (21) revealed that platelets can be redirected to exert anti-inflammatory effects on Mfs after dexamethasone-loading, suggesting this as a promising therapeutic approach to treating unresolved inflammation. In our experiment previous addition of DEX to Mfs cultures weakened the pro-inflammatory response of Mfs to PRP.

We demonstrated that the blood-derived preparations heterologous AMP extract and autologous PRP both shift Mfs towards a pro-inflammatory rather than a repair phenotype. If manipulated, proinflammatory responses must however be tightly controlled to prevent extensive damage of the host tissues. Therefore, because of the crucial role of Mfs in the regulation of inflammatory process, in cases of excessive inflammation the application of these preparations should be restricted.

Conflict of Interests Statement: The authors declare that there is no conflict of interests regarding the publication of this article.
Financial Disclosure Statement: This research was financed by the Faculty of Veterinary Medicine, University of Life Sciences in Lublin.

Animal Rights Statement: The experiments on animals were conducted in accordance with local Ethical Committee laws and regulations as regards care and use of laboratory animals.

Acknowledgements: The authors thank Kamila Łakomska and Jarosław Lisowski for technical assistance.

\section{References}

1. Agier J., Efenberger M., Brzezińska-Błaszczyk E.: Cathelicidin impact on inflammatory cells. Centr Eur J Immunol 2015, 40, 225-235.

2. Anderson R.C., Rehders M., Yu P.L.: Antimicrobial fragments of the pro-region of cathelicidins and other immune peptides. Biotechnol Lett 2008, 30, 813-818.

3. Arrepol E.G.S., Pérez C., Escobar A., Ortiz M.C., Tempio Sepúlveda F., Lopez M.N.: Whole platelets induces macrophage programming toward a repair profile characterized by high IL-10 production. Front Immunol Conference Abstract. $11^{\text {th }}$ Congress of the Latin American Association of Immunology - 100 Congreso de la Asociación Colombiana de Alergia, Asma e Inmunología. doi: 10.3389/conf.fimmu.2015.05.00015 (2015).

4. Campbell L., Emmerson E., Williams H., Saville C.R., Krust A., Chambon P., Mace K.A., Hardman J.: Estrogen receptor-alpha promotes alternative macrophage activation during cutaneous repair. J Invest Dermatol 2014, 134, 2447-2457.

5. Campbell L., Saville C.R., Murray P.J., Cruickshank S.M., Hardman J.: Local arginase 1 activity is required for cutaneous wound healing. J Invest Dermatol 2013, 133, 2461-2470.

6. Cecillio C.A., Costa E.H., Simioni P.U., Gabriel D.L., Tamashiro W.M.S.C.: Aging alters the production of iNOS, arginase, and cytokines in murine macrophages. Braz J Med Biol Res 2011, 44, 671-681.

7. Corraliza I.M., Campo M.L., Soler G., Modolell M.: Determination of arginase activity in macrophages: a micromethod. J Immunol Methods 1994, 174, 231-235.

8. Czakai K., Dittrich M., Kaltdorf M., Müller T., Krappmann S., Schedler A., Bonin M., Dühring S., Schuster S., Speth C., Rambach G., Einsele H., Dandekar T., Löffler K.J.: Influence of platelet-rich plasma on the immune response of human monocyte-derived dendritic cells and macrophages stimulated with Aspergillus fumigatus. International J Med Microbiol 2017 , 307, 95-107.

9. Eligni S., Crisci M., Bono E., Songia P., Tremoli E., Colombo G.I., Coll S.: Human monocyte-derived macrophages spontaneously differentiated in vitro show distinct phenotypes. J Cell Physiol 2013, 228, 1464-1472.

10. Elmowalid G.: A simple method for generation of functional sheep peripheral blood monocytes-derived-macrophages in vitro. Global Veterinaria 2012, 9, 590-599.

11. Giles K., Ross K., Rossi A.G., Hotchin N.A., Haslett C., Dransfield I.: Glucocorticoid augmentation of macrophage capacity for phagocytosis of apoptotic cells is associated with reduced p130 Cas expression, loss of paxillin/pyk2 phosphorylation, and high levels of active Rac. J Immunol 2001, 167, 976-986.

12. Mantovani A., Sica A., Sozzani S., Allavena P., Vecchi A., Locati M.: The chemokine system in diverse forms of macrophage activation and polarization. Trends Immunol 2004, 25, 677-686. 
13. McPhee J.B., Hancock R.E.W.: Function and therapeutic potential of host defence peptides. J Pep Sci 2005, 11, 677-687.

14. McWhorter F., Wang T., Nguyen P., Chung T., Liu W.F.: Modulation of macrophage phenotype by cell shape. PNAS 2013, 10, 17253-17258.

15. Murray P.J., Wynn T.A.: Protective and pathogenic functions of macrophage subsets. Nat Rev Immunol 2011, 11, 723-737.

16. Pelillo C., Benincasa M., Scocchi M., Gennaro R., Tossi A., Pacor S.: Cellular internalization and cytotoxicity of the antimicrobial proline-rich peptide Bac7(1-35) in monocytes/ macrophages, and its activity against phagocytosed Salmonella typhimurium. Protein Peptide Lett 2014, 21, 382-390.

17. Ploeger D.T.A., Hosper N.A., Schipper M., Koerts J.A., de Rond S. Bank R.A.: Cell plasticity in wound healing: paracrine factors of $\mathrm{M} 1 / \mathrm{M} 2$ polarized macrophages influence the phenotypical state of dermal fibroblasts. Cell Commun Signal 2013, 11, 29.

18. Porcheray F., Viaud S., Rimaniol A.C., Leone C., Samah B., Dereuddre-Bosquet N., Dormont D., Gras G.: Macrophage activation switching: an asset for the resolution of inflammation. Clin Experiment Immunol 2005, 142, 481-489.

19. Sang Y., Blecha F.: Porcine host defense peptides: expanding repertoire and functions. Develop Comp Immunol 2009, 33, 334-343.

20. Schnabel L.V., Mohammed H.O., Miller B.J., McDermott W.G., Jacobson M.S., Santangelo K.S., Fortier L.A.: Platelet rich plasma (PRP) enhances anabolic gene expression patterns in flexor digitorum superficialis tendons. J Orthop Res 2007, 25 , 230-240.

21. Scull C.M., Hays W.D., Fischer H.: Macrophage proinflammatory cytokine secretion is enhanced following interaction with autologous platelets. J Inflamm 2010, 7, 53.

22. Spiller K.L., Anfang R.R., Spiller K.J., Ng J., Nakazawa K.R., Daulton J.W., Vunjak-Novakovic G.: The role of macrophage phenotype in vascularization of tissue engineering scaffold. Biomaterials 2014, 35, 4477-4488.

23. Szponder T., Wessely-Szponder J., Świeca M., Smolira A., Gruszecki T.: The combined use of ozone therapy and autologous platelet-rich plasma as an alternative approach in foot rot treatment in sheep. A preliminary study. Small Rumin Res 2017, 156, 50-56.

24. Szponder T., Wessely-Szponder J., Smolira A. Evaluation of platelet-rich plasma and neutrophil antimicrobial extract as two autologous blood-derived agents. Tissue Eng Regen Med 2017, 14, 287-296

25. Wessely-Szponder J., Szponder T., Bobowiec R.: Different activation of monocyte-derived macrophages by antimicrobial peptides at a titanium tibial implantation in rabbits. Res Vet Sci 2017, 115, 201-210.

26. Zizzo G., Cohen P.L.: IL-17 stimulates differentiation of human anti-inflammatory macrophages and phagocytosis of apoptotic neutrophils in response to IL-10 and glucocorticoids. J Immunol 2013, 190, 5237-5246, doi:10.4049/jimmunol.1203017. 